\title{
PLANES GERONTOLÓGICOS Y PROYECTOS DE ANIMACIÓN SOCIOCULTURAL PARA LAS PERSONAS MAYORES
}

\author{
Gerontology plans and projects of "socio-cultural \\ animation" for elderly people
}

\author{
Agustín REQUEJO OSORIO \\ Universidad de Santiago de Compostela. Campus Sur. 15706 Santiago de Compostela. \\ Correo-e: hearo@usc.es
}

Fecha de aceptación definitiva: marzo de 2001

BIBLID [(1130-3743) 12, 2000, 85-105]

\section{RESUMEN}

En el contexto de una población cada vez más anciana, el presente trabajo analiza, en una primera parte, los diferentes planes gerontológicos del Estado español y de las diferentes regiones autonómicas que los han aprobado y aplicado en los últimos años.

El interés por la multiplicidad y evolución de dichos planes está relacionado con el proyecto internacional de evaluación para el año 2001 del «Plan de Acción Internacional sobre el Envejecimiento" propuesto por Naciones Unidas a lo largo de la última década.

Un aspecto particular de dichos planes - junto al tema de las pensiones, salud, asistencia sanitaria, servicios sociales y participación- se refiere a las cuestiones de cultura y tiempo libre.

Por tal motivo esta cuestión es valorada en distintos planes gerontológicos y estudiada en el contexto de los procesos y planes de animación sociocultural con las personas mayores para transformar su ocio pasivo en ocio activo mediante programas y actividades que lo fomentan.

Con carácter particular se estudian las características del ocio de las personas mayores en Galicia en algunos de estos programas (universidades populares, IV ciclo universitario) considerando su importancia para la mejor participación, desarrollo cultural y calidad de vida de las personas mayores. 
Descriptores: planes gerontológicos, animación sociocultural, ocio y tiempo libre, política social, personas mayores.

\section{SUMMARY}

Within the context of a population increasingly older, the first part of this paper analyses the different gerontology plans of the Spanish State and its autonomous regions, which approved and applied them along the last few years.

The interest for the great variety and evolution of said plans it is related to the international project on evaluation for the year 2001 of the "International Action Plan for Elderly People" proposed by the United Nations along the last decade.

A particular aspect of said plans - together with the themes of pensions, health, health aids, social services and participation - refers to the issues of culture and leisure time. Therefore, this fact is greatly valued among the various gerontology plans and it is studied within the context of the processes and plans of "socio-cultural animation" for elderly in order to transform their passive leisure time into an active one by using the programmes and activities that promote it.

Finally, and within some of these programmes [universidades populares ("popular universities", or a specific type of adult education centres), "IV ciclo universitario" (4th university cycle, or university studies for elderly people)], the particular characteristics of the leisure time of Galician elderly are studied. The relevance of these programmes for a better participation, a better cultural development and a better quality of life for elderly people is considered.

Key words: gerontological programmes, socio-cultural activities, leisure time, social politic, olders adults.

\section{LA MULTiPlicidad DE PLANES GerONTOLÓGicos}

En los últimos años a partir del Plan Gerontológico del Estado (1992) elaborado por el Inserso (Instituto Nacional de Servicios Sociales) se han multiplicado en nuestro país, por parte de las diferentes autonomías, los planes gerontológicos o planes sectoriales en favor de las personas mayores. La razón fundamental para esta atención gerontológica a las personas mayores hay que buscarla en múltiples motivos.

En primer lugar, ya desde 1982 la "Asamblea Mundial del Envejecimiento" propiciaba un "Plan de acción internacional". Proponía además un marco referencial al que debían ajustarse los diferentes estados en las próximas décadas determinando en diversos documentos y propuestas cómo poner en práctica y fijando en sucesivas resoluciones unos objetivos mundiales para el año $2001^{1}$ entre los que destacan los

1. Para este año (2001) se ha fijado, por parte de la Asamblea de Naciones Unidas, una serie de actividades: evaluación de lo que fue el Año Internacional de las Personas Mayores (1999); de las estrategias para alcanzar los objetivos fijados en 1992 y el quinto examen del Plan Internacional de 
siguientes: 1) Apoyo a los países en el establecimiento de objetivos nacionales relativos al envejecimiento; 2) Promoción de apoyo para integrar el envejecimiento en los planes y programas nacionales e internacionales de desarrollo; 3) Promoción de apoyo a los programas de atención y participación de las personas de edad más avanzada basados en la comunidad; 4) Inclusión del tema sobre el envejecimiento en celebraciones y reuniones internacionales de importancia; 5) Establecimiento de una red mundial de voluntarios de mayor edad en pro del desarrollo social y económico; 6 y 7) Promover la viabilidad de una cooperación más estrecha entre las organizaciones no gubernamentales e intergubernamentales en relación con el envejecimiento ${ }^{2}$.

En el caso español en 1992 se aprueba el primer Plan Gerontológico del Estado propiciado por el entonces Ministerio de Asuntos Sociales ${ }^{3}$. Con ello España trata de fijar sus grandes objetivos en cuanto a la población de más edad. Al mismo tiempo se pretende desarrollar una política social ajustada a los principios de la nueva Constitución (art. 40, 41, 43, 44, 47 y 49) y especialmente el referido a las personas mayores:

"los poderes públicos garantizarán mediante pensiones adecuadas y periódicamente actualizadas, la suficiencia económica de los ciudadanos durante la tercera edad. Así mismo, y con independencia de las obligaciones familiares, promoverán su bienestar mediante un sistema de servicios sociales que atenderán a los problemas específicos de salud, vivienda, cultura y ocio" (art. 50).

La propia Constitución, que plantea una España ampliamente descentralizada, es la que favorecerá que los diferentes gobiernos autonómicos elaboren progresivamente sus leyes de "Servicios Sociales" ${ }^{4}$ y también se decidan, algunos de ellos, a poner en marcha sus respectivos "Planes Gerontológicos". El plan del Estado es más de carácter indicativo que obligatorio y vinculante para las comunidades autónomas que tienen competencias en este tema 5 .

Acción que servirá de base para nuevas propuestas a nivel mundial durante el período 2010-2020. En el año 2002 se prevé por parte de Naciones Unidas celebrar en España la "II Asamblea Mundial sobre el Envejecimiento".

2. Asamblea Mundial de Naciones Unidas. Viena 1982. Publicación del Plan de "Acción Internacional. (1991). Ejecución del Plan Internacional sobre el Envejecimiento y actividades conexas. Principios básicos (1993).

3. Elaborado entre 1988-1991 se operativiza a partir de 1992 con sucesivos balances y revisiones en el momento actual para su adaptación a los retos del siglo xxi, donde el número de personas mayores, a tenor de los datos demográficos, irá aumentando.

4. A lo largo de la década 1982-1992 un importante número de autonomías han aprobado en sus respectivos parlamentos las Leyes de Servicios Sociales: País Vasco (1982) reformada en 1996; Navarra (1983); Madrid (1984); Murcia (1985); Cataluña (1985) reformada en 1994; Castilla-La Mancha (1986); Islas Baleares (1987); Aragón (1987); Islas Canarias (1987); Asturias (1987); Galicia (1987) reformada en 1993; Andalucía (1988); Castilla y León (1988); Valencia (1989); La Rioja (1990) y Cantabria (1992).

5. Los diferentes planes gerontológicos aparecen a lo largo de la presente década 1990-2000: País Vasco (1990) y (1994); Castilla y León (1995); Islas Baleares (1996); Navarra (1997); Castilla-La Mancha (1998); Madrid (1998). Por el momento solamente algunas comunidades han estimado conveniente su 
En segundo lugar, el interés por el desarrollo tanto de leyes específicas de Servicios Sociales como de "planes gerontológicos" viene propiciado por la importancia que el colectivo de personas mayores está adquiriendo tanto desde el punto de vista demográfico, como sociopolítico.

En el ámbito demográfico es de sobra conocido que Europa está envejeciendo de forma progresiva. Los diagnósticos del futuro más inmediato (los próximos 25 años) no son muy optimistas: "la Unión Europea se enfrenta al siglo xxi con una población activa más reducida y envejecida y con una cantidad mayor de personas mayores y jubiladas" ${ }^{6}$. El caso de España es particularmente significativo ya que se están acrecentando en nuestro país las tendencias europeas. El crecimiento sólo ha sido del 0,46\% en el período 1996-1998. Su aumento ha sido un poco más positivo del 1,05\% en el bienio 1998-2000 superándose la cifra de los cuarenta millones. Con todo, según los datos estimados de la onu, es posible que nuestro país, si no cambian las tendencias demográficas, pierda habitantes en los próximos cincuenta años debido a la disminución de la tasa de natalidad?

En el contexto sociopolítico los mayores comienzan a representar unos de los colectivos más importantes como fenómeno social que afecta a las cuestiones laborales, a las pensiones, a la planificación y atenciones sanitarias y, evidentemente, a la vida política en general donde su capacidad de decisión mediante el voto está cobrando una relevancia importante ${ }^{8}$.

Desde estas coordenadas, presentadas de forma sintética, se puede entender que las instancias políticas y sociales se hayan dedicado en los últimos años a proponer análisis, articular respuestas y sobre todo a plantear acciones políticas más o menos concretas en distintas órdenes.

Son estas últimas las que brevemente pretendemos analizar en este texto para centrarnos fundamentalmente en un apartado más específico: los proyectos de animación

realización y puesta en acción. Otras han preferido proponer planes sectoriales como es el caso de Cataluña: "Promoció dels drets $i$ la vida activa de les persones grans: Plan integral de la gent gran: Noves iniciatives i programes". (1995) y Galicia: "Plan general de actuación con las personas mayores" (1998).

A su vez otras autonomías han elaborado sus correspondientes legislaciones: Canarias (Ley 3/1996) sobre "Participación de las personas mayores y de la solidaridad entre la generaciones"; Andalucía: Ley 6/1999 de "Atención y protección a las personas Mayores".

6. Para los últimos datos publicados (1996) un 23,7\% de la población tenía menos de 20 años y un 20,8\% era mayor de 60 años siendo el 3,9\% el conjunto de personas mayores de 80 años.. Cfr. EurosTAT (1999): Europa en cifras. Mundi Prensa, Madrid, pp. $46-56$.

7. En concreto según el censo del 2000 publicado por el Instituto Nacional de Estadística se alcanzó la cifra de 40.202 .160 habitantes. A pesar de este ligero incremento el último informe de Naciones Unidas no es nada optimista para España. Para el año 2050 calcula una cifra de 30.226 .000 habitantes, lo que supondría una pérdida de población muy alta: 9.976.160. La media de edad del conjunto de la población sería muy alta (54,3 años).

8. Basta simplemente con consultar las múltiples polémicas mantenidas sobre el problema de las "pensiones" entre los dos grandes partidos con motivo de las últimas elecciones generales (El País, marzo 2000). 
sociocultural referidos de forma concreta a las cuestiones de ocio y tiempo libre de las personas mayores.

\subsection{Política social con las personas mayores: del Plan Gerontológico Estatal al desarrollo de algunos planes autonómicos}

El análisis de las personas mayores tiene el riesgo del alarmismo demográfico, ciertamente no infundado, pero olvidándose de la intervención social para atender a este colectivo. Su problema no es un problema sólo de número, es mucho más: se trata de un problema que tiene una dimensión social. En otros términos, no podemos limitarnos a analizar y discutir las cifras demográficas por muy espectaculares y problemáticas que se nos presenten.

Es necesario avanzar hacia una consideración de las personas de más edad como fenómeno social entendido no sólo a partir de los gastos que puedan generar (sanitarios, asistenciales, de pensiones, etc.) sino como una cuestión de reconocimiento de su papel social. El objetivo no es por tanto una consideración asistencial de las personas mayores sino permitir que todas las personas, particularmente las de edad más avanzada, desempeñen su propio papel en la sociedad y asuman libremente sus deberes y derechos como ciudadanos en todas las edades.

Todo ello supone proponer y mantener una política social desarrollada y configurada en el denominado "Estado del bienestar" que estimule y apoye a sus ciudadanos a favor de la solidaridad y cohesión social asegurándoles una serie de prestaciones por medio de los correspondientes servicios sociales.

Estos servicios sociales se estructuran hoy en base a dos modalidades: servicios sociales comunitarios que configuran la estructura básica del sistema ofertados a toda la población y apoyados básicamente desde la Administración municipal. Los servicios sociales especializados (como segundo nivel de intervención) que van dirigidos a determinados sectores de población que por sus condiciones requieren una atención específica como es el caso de las personas mayores.

El presente y futuro de estas políticas sociales va a estar determinado por diferentes condicionantes: uno de carácter más estructural y otro de carácter más político e ideológico.

En el primer caso, el fenómeno estructural más acuciante es el incremento demográfico de las personas de más edad, tal como se ha expuesto anteriormente, junto con los cambios y transformaciones laborales que se están produciendo. Nos referimos en este caso a un grupo de mayores (aquellos que se encuentran en la denominada "adultez intermedia" a partir de los 50 años) cuyas tasas de actividad están descendiendo en la última década en los países desarrollados forzándoles a un abandono precoz del mercado laboral en base a múltiples causas (ajustes de plantillas, fusiones de empresas, sustitución de mano de obra a un menor coste, etc.).

En el segundo caso, los determinantes de esta política social están relacionados con cuestiones más de carácter ideológico-político. En opinión de G. Rodríguez 
Cabrero 9 existen dos modelos básicos de enfoque de políticas para las personas mayores. El modelo ideológico escorado hacia el campo del alarmismo producto de los datos estadísticos que nos llegan de los censos. Desde este modelo se instauran políticas meramente asistenciales o, a veces, de exclusión basadas en la institucionalización, la rigidez de la jubilación cuando no su anticipación, las políticas de renta y subsistencia, la yuxtaposicón de medidas con poca integración, etc.

El modelo sociohistórico, por su parte, considera que los mayores son una construcción social y no sólo el proceso natural del envejecimiento. Por tanto un grupo con una amplia diferenciación que está socialmente estructurado y en consecuencia abierto al cambio. Desde esta interpretación, las políticas sóciales que se realicen pueden jugar un papel ambivalente: pueden crear tanto dependencia como bienestar.

En el caso que aquí más nos interesa pueden ocasionar falta de participación, desinterés social o bien adaptación y transformación en lo referente a la práctica del ocio, motivación por la formación, compromiso social de participación y ayuda al cambio social.

La intención de Plan Gerontológico Estatal, como la de los planes de diversas comunidades, surge con la idea fundamental de promover una política social que ayude a comprender la vida y las potencialidades de las personas mayores exigiendo un cambio de actitudes; de asegurar la igualdad de oportunidades; de garantizar las necesidades básicas y recuperar a los mayores como fuerza social al mismo tiempo que se contribuye a su bienestar.

En cuanto al Plan Gerontológico del Ministerio hay que decir que su gran finalidad era fijar un marco de desarrollo armónico de una política social relacionada con las personas mayores en cinco ámbitos específicos: 1) Pensiones; 2) Salud y Asistencia Sanitaria; 3) Servicios Sociales; 4) Cultura y Ocio; 5) Participación.

¿Qué ha sucedido en los últimos años con carácter general y de forma particular en la cuestión que aquí específicamente nos interesa como es el tema más cultural? Tomando como referencia la evaluación realizada por el propio Inserso (1998) éstos son algunos de los principales datos considerados en sus aspectos más esenciales. No es intención de este trabajo hacer un estudio detallado de los distintos temas y problemas gerontológicos ${ }^{10}$.

En la denominada "Área de Pensiones" se han cumplido en gran parte los grandes objetivos. Se ha desarrollado el plan de pensiones no contributivas de jubilación, la equiparación de las pensiones mínimas contributivas para mayores de 65 años sin recursos suficientes y con cónyuge a su cargo; la revalorización automática de las pensiones. Otros temas quedan todavía pendientes: la subida de su

9. Rodríguez Cabrero, G. (1997): Participación social de las personas mayores. Ministerio de Trabajo y Asuntos Sociales, Madrid, pp. 19 y ss.

10. Miguel, J. A. (1998): "Balance de las políticas sociales con los mayores en España. Los Planes Gerontológicos. Análisis y perspectivas». Documentación Social, 112, 175-191. 
importe para las más bajas y garantizar para el futuro su estabilidad dados los problemas que a corto plazo se derivarán de los cambios demográficos actuales.

Respecto a la "Salud y Asistencia Sanitaria" la atención primaria y especializada a las personas mayores alcanza unos niveles más que aceptables tras la correspondiente reordenación de los servicios sanitarios y la gratuidad de las prescripciones farmacéuticas. Esta práctica comienza a cuestionarse en los últimos meses. Donde se notan unas ciertas carencias es en la disponibilidad de las prestaciones de rehabilitación que son mucho más prolongadas en tiempo y con mayor coste cuando se trata de personas más ancianas.

En el "Área de Servicios Sociales" existen limitaciones en aspectos muy puntuales. No se ha logrado el promedio de 3,5 plazas residenciales por cada cien personas mayores tal como se recomendaba. La media de todas las comunidades autónomas está en el 2,83. Algunas comunidades como Galicia (1,52), Murcia (1,96), Madrid $(2,27)$ están un poco distantes de dicho objetivo. Importante ha sido el aumento del número de hogares, clubs e instituciones similares, pero su distribución en poblaciones no urbanas o de tamaño medio resulta insuficiente.

Por lo que respecta a la cuestión de la "Participación" se han incrementado notablemente las asociaciones de personas mayores en los últimos años. En 1994 se ha creado el Consejo Estatal de las Personas mayores ${ }^{11}$ pero todavía queda una cierta "inercia" a depender más de los servicios que a ser sujetos activos en defensa de los derechos que corresponden a este colectivo.

Por último, en el tema de "Cultura y Tiempo Libre» la situación resulta más problemática como luego veremos con más detalle. Es cierto que hay un mayor auge de iniciativas culturales y educativas pero, tal como se dice en el informe de evaluación del Plan Gerontológico, "el ocio de nuestros mayores no se orienta hacia la realización de actividades con ese contenido, quizá como secuela de las reducidas posibilidades de enriquecimiento educativo y cultural de que gozaron las actuales personas mayores en su juventud". De hecho los grandes programas de ocio tienen mucho que ver con el "turismo". En los últimos años esta actividad se ha complementado con las ofertas "termalismo" 12 .

11. Dicho consejo es un órgano colegiado de carácter consultivo para la Administración General del Estado, adscrito al Ministerio de Trabajo y Asuntos Sociales. Sus funciones generales son, entre otras, las siguientes: 1) Asesorar sobre las convocatorias efectuadas por los órganos de la Administración del Estado dirigidas a asociaciones de personas mayores; 2) Seguimiento y aplicación del Plan Gerontológico Nacional; 3) Proponer, en función de la necesidad y oportunidad, estudios e investigaciones sobre la situación y calidad de vida de los mayores.

12. Ibídem, p. 187. El mayor número de actividades y de plazas se dieron en estos contextos. Conviene recordar que nuestro país, como segundo país mundial en la recepción de turistas, cuenta con numerosas instalaciones que en los meses de octubre-mayo ofrecen sus servicios a las personas mayores. De esta forma pueden viajar y acogerse a unos días de vacaciones en los múltiples lugares que ofrece el territorio español. En el año 1997, participaron aproximadamente en el Programa de "Vacaciones" 360.000 personas mayores. En el caso del Programa de "Termalismo", donde además del turismo se une la función terapéutica que cumplen los baños para determinadas enfermedades o dolencias, la participación fue de 65.000 . 


\subsection{Los planes gerontológicos de carácter autonómico}

Siguiendo las pautas del plan estatal, diversas autonomías han ido elaborando y poniendo en marcha sus propios planes gerontológicos. Tienen un carácter más puntual y ajustado a la situación de cada una de ellas, analizando con todo detalle de datos la mayoría de temas y cuestiones que preocupan a las personas mayores.

A partir de este estudio detallado señalan a continuación los grandes objetivos y programas y/o planes de actuación. Todos ellos tienen en común tres grandes temas: en primer lugar, el diagnóstico de la evolución de la población mayor que en muchos casos incluye el estudio sobre la situación de su vida; su actividad, la participación y ocio; los recursos y la atención a los mayores. En segundo lugar, los principios y objetivos que se proponen en dicho plan. Finalmente estrategias o planes de actuación en los diferentes campos promoviendo, en unos casos, modelos de atención y en otros hablando de estrategias de actuación: programas y proyectos.

Dada la amplitud de estos documentos, simplemente se pretende resaltar aquí una enumeración general los principales objetivos y programas para luego centrarnos en el análisis más específico del tema del ocio y tiempo libre.

En síntesis, los principales objetivos que estos planes se proponen discurren por cuatro diferentes preocupaciones a tenor de las indicaciones más generales que cada uno de ellos enumera de forma particular (cfr. tabla 1):

- Participación, integración, presencia en el entorno y cohesión social respecto al colectivo de mayores.

- Consideración positiva de esta etapa de vida, de sus valores para afrontarla con un sentido positivo y de desarrollo a lo largo de la vida.

- Especial ayuda (información, orientación, apoyo, etc.) a los mayores dependientes, incapacitados, asegurándoles los servicios necesarios.

- Promoción del ocio y cultura que, si bien aparece de forma minoritaria en este apartado de objetivos, se asume como capítulo aparte en algunos planes, tal como luego indicaremos y estudiaremos con más detalle. 
TABLA No 1. Principales objetivos de los planes gerontológicos

\begin{tabular}{|c|c|c|c|c|}
\hline CASTILLA-LA MANCHA & CASTILLA Y LEÓN & NAVARRA & MADRID & PAIIS VASCO \\
\hline $\begin{array}{l}\text { 1. Potenciar los } \\
\text { servicios de } \\
\text { información, } \\
\text { valoración y } \\
\text { orientación a las } \\
\text { personas } \\
\text { mayores. }\end{array}$ & $\begin{array}{l}\text { 1. Sensibilizar a la } \\
\text { sociedad hacia } \\
\text { una actitud } \\
\text { positiva y realista } \\
\text { de la tercera edad. }\end{array}$ & $\begin{array}{l}\text { 1. Mantener y fomen- } \\
\text { tar la cohesión } \\
\text { social evitando el } \\
\text { alejamiento de las } \\
\text { generaciones y } \\
\text { procurando el } \\
\text { reconocimiento de } \\
\text { sus valores. }\end{array}$ & $\begin{array}{l}\text { 1. Promover una } \\
\text { vivencia positiva } \\
\text { de la última etapa } \\
\text { vital. }\end{array}$ & $\begin{array}{l}\text { 1. Posibilitar a los } \\
\text { ancianos llevar } \\
\text { una vida } \\
\text { independiente en } \\
\text { el seno de su } \\
\text { propia } \\
\text { comunidad. }\end{array}$ \\
\hline $\begin{array}{l}\text { 2. Garantizar la } \\
\text { protección } \\
\text { económica a } \\
\text { todos los } \\
\text { mayores en } \\
\text { situación de } \\
\text { necesidad. }\end{array}$ & $\begin{array}{l}\text { 2. Apoyar a las } \\
\text { personas mayores } \\
\text { para que afronten } \\
\text { de manera } \\
\text { positiva la etapa } \\
\text { de jubilación y } \\
\text { puedan continuar } \\
\text { su desarrollo } \\
\text { personal a lo } \\
\text { largo de la vida. }\end{array}$ & $\begin{array}{l}\text { 2. Favorecer la } \\
\text { integración y } \\
\text { participación social } \\
\text { de personas que } \\
\text { en virtud de su } \\
\text { edad quedan } \\
\text { excluidas del } \\
\text { mundo laboral } \\
\text { fomentando la } \\
\text { utilización de } \\
\text { nuevos cauces de } \\
\text { participación. }\end{array}$ & $\begin{array}{l}\text { 2. Prevenir los riesgos } \\
\text { asociados a la edad } \\
\text { susceptibles de } \\
\text { hacer a las personas } \\
\text { vulnerables. }\end{array}$ & $\begin{array}{l}\text { 2. Abordaje } \\
\text { multisectorial de } \\
\text { sus problemas } \\
\text { garantizando una } \\
\text { asistencia } \\
\text { sanitaria y } \\
\text { desarrollando los } \\
\text { servicios sociales } \\
\text { que fomentan la } \\
\text { autovalidez del } \\
\text { anciano y } \\
\text { mantenimiento } \\
\text { en su entorno. }\end{array}$ \\
\hline $\begin{array}{l}\text { 3. Facilitar la } \\
\text { movilidad y } \\
\text { accesibilidad al } \\
\text { medio físico de } \\
\text { las personas } \\
\text { mayores. }\end{array}$ & $\begin{array}{l}\text { 3. Incrementar la } \\
\text { autonomía perso- } \\
\text { nal y bienestar } \\
\text { psicosocial de los } \\
\text { mayores promo- } \\
\text { viendo las activi- } \\
\text { dades de ocio y } \\
\text { cultura, así como } \\
\text { su participación } \\
\text { dentro de su } \\
\text { entorno y de la } \\
\text { sociedad en } \\
\text { general. }\end{array}$ & $\begin{array}{l}\text { 3. Fomentar el } \\
\text { mantenimiento de } \\
\text { su autonomía } \\
\text { ofreciéndoles } \\
\text { medios para } \\
\text { desarrollar sus } \\
\text { potencialidades y } \\
\text { frenar los } \\
\text { procesos de } \\
\text { involución que } \\
\text { acompañan a la } \\
\text { edad avanzada. }\end{array}$ & $\begin{array}{l}\text { 3. Atender las } \\
\text { situaciones } \\
\text { producidas de } \\
\text { precariedad y } \\
\text { necesidades de } \\
\text { recursos en las } \\
\text { personas mayores. }\end{array}$ & $\begin{array}{l}\text { 3. Fomentar la } \\
\text { participación de } \\
\text { los profesionales } \\
\text { en la atención del } \\
\text { anciano. }\end{array}$ \\
\hline $\begin{array}{l}\text { 4. Garantizar la } \\
\text { defensa de los } \\
\text { mayores } \\
\text { incapacitados } \\
\text { legalmente o en } \\
\text { situación de } \\
\text { desamparo. }\end{array}$ & $\begin{array}{l}\text { 4. Favorecer la } \\
\text { permanencia de } \\
\text { las personas } \\
\text { mayores en su } \\
\text { medio ambiente } \\
\text { habitual. }\end{array}$ & $\begin{array}{l}\text { 4. Potenciar la } \\
\text { asunción de } \\
\text { corresponsabiliclad } \\
\text { social en atención } \\
\text { a los individuos } \\
\text { dependientes. }\end{array}$ & $\begin{array}{l}\text { 4. Fomentar la } \\
\text { integración y } \\
\text { participación de } \\
\text { los mayores en } \\
\text { sociedad. }\end{array}$ & $\begin{array}{l}\text { 4. Desarrollar la } \\
\text { concienciación de } \\
\text { la sociedad acerca } \\
\text { de sus } \\
\text { necesidades y la } \\
\text { problemática que } \\
\text { les aqueja para } \\
\text { dotarles de un } \\
\text { mayor nivel de } \\
\text { integración } \\
\text { posible. }\end{array}$ \\
\hline $\begin{array}{l}\text { 5. Asegurar un } \\
\text { adecuado nivel } \\
\text { de calidad en } \\
\text { centros y } \\
\text { servicios sociales } \\
\text { para mayores. }\end{array}$ & $\begin{array}{l}\text { 5. Garantizar a las } \\
\text { personas un } \\
\text { alojamiento digno } \\
\text { y adaptado a sus } \\
\text { necesidades. }\end{array}$ & $\begin{array}{l}\text { 5. Favorecer la pre- } \\
\text { sencia de los } \\
\text { mayores en su } \\
\text { entorno social. }\end{array}$ & & $\begin{array}{l}\text { 5. Estimular la } \\
\text { participación de } \\
\text { los ancianos en las } \\
\text { actividades } \\
\text { sociales. }\end{array}$ \\
\hline
\end{tabular}


Junto a estos grandes objetivos como filosofía de acción básica, los propios planes coinciden en aspectos más puntuales: asegurar un adecuado nivel de calidad en centros y servicios sociales para mayores, atender particularmente a personas excluidas del mundo laboral, protección económica de los más necesitados, participación de los profesionales en la atención del anciano, etc.

En el tema del ocio y tiempo libre la mayoría de los planes dedican un apartado específico, más o menos amplio, a esta problemática. Algunas comunidades realizan un diagnóstico de la situación (Navarra, Castilla-La Mancha y Madrid). Otras, como Castilla y León, sugieren algunas propuestas mientras que el País Vasco no dedica un apartado específico a este tema. Simplemente se contenta con destacar que "existe escasez de oferta de alternativas destinadas al aprovechamiento del ocio y tiempo libre de las personas mayores".

Resumiendo de forma sintética los análisis presentados por algunos de los diferentes planes gerontológicos, éstos son sus resultados:

A) En cuanto a Navarra y su diagnóstico de la situación se recogen las principales opiniones:

- La mayor tendencia es hacia un "ocio pasivo" aunque la situación está cambiando. El mayor porcentaje se dedica a ver la televisión o escuchar la radio, descendiendo los que se dedican a la lectura y trabajos manuales.

- La actividad principal de las personas mayores sigue siendo "pasear y charlar". Pocos son los que practican deporte: sólo la gimnasia y natación tiene alguna cuota de significación, fundamentalmente entre las mujeres.

- Crece el deseo de participar y ha aumentado el nivel de asociacionismo en los últimos años.

- Las actividades preferidas son las excursiones (Navarra, pp. 33-35).

B) Por lo que respecta a Castilla-La Mancha, se exponen las siguientes consideraciones:

- Se destaca la poca participación de las personas mayores en las actividades de ocio y tiempo libre, debido fundamentalmente a las siguientes causas: la pérdida de valor social en la cultura moderna de las actividades no remuneradas y, por tanto, aumento del sentimiento de inutilidad; la ruptura que supone el fin de la vida laboral sin una preparación ni alternativa de ocupación del tiempo; la pérdida de interés en la sociedad y, por tanto, la disminución en la participación social en general dependiendo fundamentalmente del nivel cultural y de la situación económica de las personas mayores.

- En todo caso las mujeres tiene menos problemas de adaptación que los hombres, necesitados de actividades alternativas con las que llenar 
el tiempo de ocio que deja el fin de la vida laboral (Castilla-La Mancha, pp. 25 y 26 ).

C) Finalmente la Comunidad Autónoma de Madrid ofrece algunos datos estadísticos que tendremos en cuenta en un breve estudio comparado al final de este trabajo:

- Se destaca la poca participación de las personas mayores en las actividades de ocio y tiempo libre, debido fundamentalmente a las siguientes causas: la ruptura que supone el fin de la vida laboral sin una alternativa de la ocupación del tiempo; la pérdida de interés en la sociedad y, por tanto, la disminución en la participación social en general y, por último, el nivel cultural y la situación económica de las personas mayores.

- Aunque no están carentes de relaciones sociales, las actividades cotidianas de la mayoría se reducen, más allá de las tareas domésticas, a pasear, ver la televisión y oír la radio (Madrid, pp. 26 y ss.).

En resumen, todos los planes gerontológicos regionales detectan importantes problemas en cuanto a ocio y tiempo libre de las personas mayores y ello nos obliga de alguna forma a matizar estas opiniones y a prestar una particular atención en el siguiente apartado.

Mientras tanto el plan gerontológico como los respectivos planes autonómicos han puesto de relieve la necesidad de mantener y renovar en todo el estado un marco técnico, estable, homogéneo, coordinado y equitativo en lo que se refiere a los servicios y prestaciones sociales dirigidos a las personas mayores.

Para a un futuro más inmediato, a tenor de la evaluación realizada cara al 2001, parece necesario insistir en la prevención y promoción de un envejecimiento satisfactorio para los mayores, en su participación real y efectiva en la configuración de programas y servicios a ellos dirigidos y en la toma de decisiones que les afecten, en la atención a la situación económica de los más dependientes, en el desarrollo de infraestructuras (residencias). Su escasez es evidentemente manifiesta. A tenor del estudio presentado por la Federación Nacional de Médicos de Residencias bajo el título de "Medicina Geriátrica en Residencias" " ${ }^{13}$ tenemos en España 6.330 .373 personas mayores de 65 años (15,98\%). Los centros de titularidad pública son 841 con 58.493 plazas (30,97\%). Los centros de titularidad privada son 2.832 con 130.369 plazas $(69,03 \%)$. Las diferencias son bastante significativas a la hora de prestar estos servicios.

Finalmente parece necesario realizar una política más activa y coordinada a la hora de su participación en las cuestiones que les preocupan y facilitar un mejor empleo del ocio y del tiempo libre.

13. Cfr. Informe sobre "Medicina Geriátrica en Residencias". Resumen en El País, 18 de octubre de 2000, p. 38. 


\section{LA ANIMACIÓN SOCIOCULTURAL EN Y CON LAS PERSONAS MAYORES}

El tema de la "animación sociocultural" (ASC) ha ido cobrando interés particular en España en los últimos años en relación con las personas adultas y las personas mayores ${ }^{14}$. Por animación sociocultural entendemos un tipo de acción comunitaria que tiene como propósito principal promover en las personas y los grupos una actitud de participación activa en el proceso de su propio desarrollo. Abarca por tanto una gama muy amplia y diversa de intervenciones, programas e instituciones: centros cívicos, casas de cultura y ateneos populares, clubs de educación en el tiempo libre, organización de fiestas populares, programas de dinamización cultural, etc.

En el caso de las personas mayores la ASC surge en respuesta a una ausencia o disminución de su actividad y de sus relaciones sociales. Para colmar este posible vacío o pérdida, la ASC con los mayores tiene como objetivo fundamental la idea de integración y participación voluntaria en tareas colectivas en las que la cultura juega un papel estimulante.

Una de las funciones clave de la ASC consiste en que las personas y los colectivos se conviertan en agentes y protagonistas de su propio desarrollo. Lo que particularmente interesa en los procesos de animación es generar procesos de participación creando espacios para la comunicación de los grupos y de las personas con vistas a estimular a los diferentes colectivos a emprender procesos de desarrollo social (respuesta a sus necesidades en su espacio, tiempo, situaciones concretas) y cultural (construyendo su propia identidad colectiva, generando y participando en los diferentes proyectos y actividades culturales).

En el caso concreto de las personas mayores, nos encontramos ante un colectivo que tiene unas características muy específicas: edad, jubilación y por tanto está liberado de un trabajo determinado de forma sistemática; diferentes formas de convivencia (en pareja, viudedad, soledad ${ }^{15} \ldots$ ); situaciones de salud general y condiciones físicas muy diferenciadas; contexto residencial de acuerdo con demandas particulares; una mayor disponibilidad del tiempo libre, etc.

14. REQUejo OSORIO, A. (1997): "Animación sociocultural y Educación de Adultos" y "Animación Sociocultural en la Tercera Edad. En TRILlA, J. (coord.): Animación sociocultural: Teorías, programas y ámbitos. Ariel, Barcelona, cap. 12 y 13, pp. 239-268.

15. El tema de la soledad es uno de los problemas más preocupantes. A tenor de los diferentes planes gerontológicos obtenemos los siguientes datos en sus respectivas encuestas: en Navarra el 6\% de los hombres mayores viven solos mientras la cifra se eleva al 17\% en el caso de las mujeres (p. 28). En Castilla y León el 12,5\% de los que residen en viviendas particulares están solos. Es mucho mayor el número de mujeres que viven solas con un diferencial de 13 puntos respecto a la proporción de hombres (p. 68). Finalmente en la Comunidad de Madrid también el tema de la soledad sigue siendo predominantemente femenino: de cada 100 ancianos que viven solos, 86 son mujeres y 14 varones. Esta soledad está relacionada con el estado de viudedad principalmente (56\% de mayores eran viudas, entre las mujeres, y $22 \%$ viudos, entre los hombres) (p. 28). 
Por todo ello, los programas de Asc deben ser muy diferentes y adaptados a la situación del grupo y sus necesidades respectivas. Con carácter general se pueden establecer como objetivos básicos los siguientes: posibilitar a este colectivo su realización personal, la comprensión de su entorno y la participación en la vida comunitaria; conseguir una mayor integración en la sociedad a fin de que se oiga y valore su voz y se tengan en cuentan sus opiniones; fomentar la educación y la formación permanente; ofrecer la posibilidad de disfrutar de la cultura; establecer los cauces para que los conocimientos sean compartidos de una manera flexible, enriquecedora y amena; desarrollar actitudes críticas ante la vida mediante la animación de grupos de reflexión y debate; posibilitar la apertura a otros grupos de edad; propiciar y crear actitudes y medios para gozar de la vida plenamente.

Esta serie de objetivos generales pueden tener acogida en múltiples programas, momentos y proyectos institucionales. De manera particular y para el caso del ocio y del tiempo libre nos referiremos posteriormente a dos experiencias diversas: las universidades populares y el denominado "IV ciclo universitario" de la Universidad de Santiago de Compostela ${ }^{16}$.

\subsection{Ocio y tiempo libre: algunas consideraciones conceptuales}

Cuando hablamos de "ocio" y de "tiempo libre" los usamos con frecuencia de forma indistinta y a veces como realidades que fuesen de carácter secuencial. El ocio en este caso no sería más que una consecuencia del "tiempo libre" del que disponemos. De hecho cualquier actividad de ocio se realiza siempre dentro del tiempo libre, si bien no toda actividad realizada dentro del tiempo libre es siempre ocio. En principio ${ }^{17}$, podemos distinguir de forma sintética entre el tiempo de trabajo (que es obligatorio e incluye todo la actividad laboral de la que muchas personas mayores están ya exentas por motivos de jubilación) y el tiempo de no trabajo sujeto a otras obligaciones (atender satisfacciones humanas primarias: alimentación, higiene, descanso...) y a la solución de compromisos ineludibles de nuestra vida social (encuentros familiares, reuniones de vecinos, etc.).

Dentro del tiempo de no trabajo se encuentra el tiempo liberado de toda obligación que se destina al relax, a la distensión física y mental, al descanso pasivo o al esparcimiento cultural y el ocio propiamente tal como tiempo lleno de actividades culturalmente activas y que suele estar ligado a las aficiones personales. Por lo tanto, cuando hablamos propiamente de ocio nos referimos al tiempo libre que sirve para enriquecer nuestra personalidad y que tiene un sentido ampliamente

16. Un estudio más detallado para estas instituciones fue realizado en trabajos anteriores: Cfr. Requejo, A. (1999): “Educación de adultos: Programas Universitarios para personas mayores». Revista Adaxe, 14-15, 109-130.

17. Llull PeNialba, J. (1999): Teoria y práctica de la educación en el tiempo libre. Editorial ccs, Madrid, pp. 20 y-ss. 
formativo para nuestras vidas. Nos alejamos así del sentido peyorativo de la "ociosidad" en donde pasamos el tiempo "sin saber nada que hacer". Se trata del tiempo libre "estéril" (pasivo, tedioso, aburrido y frustrante) o, en término coloquiales, la actitud vital que rodea esta situación es la de "matar el tiempo", cuyo ejemplo más señalado es el de quedarse viendo la televisión "sin esperar nada" en absoluto.

Tal como afirma J. Trilla:

«el ocio es aquella actividad que encierra valor en sí misma, resulta interesante y sugestiva para el individuo y suele implicar una cierta motivación hacia la acción. El ocio es, por tanto, una forma positiva de emplear el tiempo libre, que el sujeto elige autónomamente y después lleva efectivamente a la práctica ${ }^{18}$.

Desde una lectura más clásica, a modo de resumen, conviene recordar la definición de J. Dumazedier:

«el ocio es el conjunto de ocupaciones a las que el individuo puede entregarse de manera completamente voluntaria tras haberse liberado de sus obligaciones profesionales, familiares y sociales para descansar, para divertirse, para desarrollar su información o su formación desinteresada o para participar voluntariamente en la vida social de su comunidad ${ }^{19}$.

En consecuencia, las tres dimensiones que parecen ser elementos fundamentales del fenómeno que denominamos ocio son las siguientes: a) disponibilidad del tiempo libre, es decir, tiempo "liberado" de nuestras obligaciones profesionales, familiares y sociales; b) actitud personal frente a un tiempo desinteresado, satisfactorio, al que nos entregamos de forma voluntaria; c) conjunto de ocupaciones o prácticas de actividades que aparecen condensadas en las tres "d": descanso, diversión y desarrollo ${ }^{20}$.

\subsection{El tiempo libre de las personas mayores: entre el "ser" y el "deber ser"}

Ya hemos visto anteriormente la valoración que hacen algunos planes gerontológicos sobre el tiempo libre de las personas mayores. Como punto de partida la visión era bastante crítica al atribuirles un "ocio pasivo", escasa participación en actos culturales, y centrados en otras actividades más bien limitadas (pasear, charlar, ver la televisión, escuchar la radio, etc.). La situación de hecho, del "ser", no parece ser muy optimista.

En estos momentos creemos que se está dando un cierto giro. En efecto, las políticas que pretenden llevar adelante en España los planes gerontológicos se

18. Trulla, J. (1993): Otras educaciones. Animación Sociocultural, formación de adultos y ciudad educativa. Anthropos, Barcelona, p. 93.

19. Dumazedier, J. (1971): Ocio y sociedad de clases. Fontanella, Barcelona, p. 20.

20. Llull Peñalba, J. (1999): o. cit, p. 26. 
están orientando por elementos de referencia distintos del pasado. Se trata, en primer lugar, de cambiar la mentalidad sobre la situación de la "vejez" frente a las tendencias "edistas" con su teoría del deterioro o la incapacidad para aprender. Afirmar, en segundo lugar, lo que de forma sintética se debatía en la Asociación Europea para la Educación de Personas Adultas (E.A.E.A.) ${ }^{21}$ : reconceptualizar la intervención educativa y social con las personas mayores desde la perspectiva de "acciones de capacitación" frente a acciones puntuales de "asistencia". Ello requiere tanto estrategias educativas como apoyos sociales para que este colectivo pueda tomar iniciativas, tener confianza en sí mismo y conciencia de su valor personal. En el fondo se trata de invertir la doble mentalidad: la de los propios mayores y la de la sociedad expresada de forma práctica en el título de la siguiente obra: ¿Qué bacen las personas mayores por nosotros? ${ }^{22}$. Es decir, contraponerla a la actitud más pasiva y asistencial: "qué hacemos nosotros por las personas mayores». En este sentido es necesario el aprovechamiento del tiempo libre entendido no como problema sino como oportunidad de aprendizaje en su sentido amplio y como una fase de desarrollo personal cargada de posibilidades.

Esto supone que es necesario para todos, pero sobre todo para las personas mayores, una integración de los tres tipos del "tiempo libre": el tiempo libre de obligación que aporta relax y diversión...; el "tiempo de formación" que genera nuevas pautas de curiosidad, de interés por los problemas de hoy, y finalmente el «tiempo social" que facilita el encuentro, las relaciones sociales pero también las oportunidades de servicio a la sociedad mediante actividades diversas de voluntariado.

\section{LAS ACTIVIDADES DE LAS PERSONAS MAYORES EN EL TIEMPO LIBRE}

Hasta el presente momento hemos tratado de analizar el planteamiento político-social de los planes gerontológicos y sus consideraciones particulares sobre algunos problemas de las personas mayores, particularmente las cuestiones de ocio y tiempo libre. En la segunda parte hemos indicado las funciones que puede cumplir la animación sociocultural en el colectivo de personas mayores y la importancia que puede tener para este colectivo el mucho tiempo libre de que dispone.

En este tercera parte se ofrecen algunos datos empíricos derivados de un trabajo de investigación en marcha sobre programas educativos para personas mayores.

Nos vamos a ceñir aquí a dos ámbitos institucionales: universidades populares ${ }^{23}$ y el denominado "IV ciclo universitario» que es un programa específico de formación

21. European Association for the Education of Aduts (1994): Older adults as helpers in learning processes. E.A.E.A. Monografías, Barcelona.

22. Domínguez Angulo, J. (1998): ¿Qué bacen las personas mayores por nosotros? Ediciones Delfín, Madrid.

23. Surgen a finales del siglo XIX y se desarrollan por toda España al igual que en otros países europeos. En la época de la dictadura franquista (1939-1975) son suprimidas pero se organizan de nuevo a partir de los ayuntamientos democráticos constituidos en 1978. En Galicia son cuatro las que 
que la propia Universidad de Santiago propone para las personas mayores de 55 años 24 .

En el caso de las universidades, a tenor de los datos ofrecidos por la Federación Española de Universidades Populares (F.E.U.P.), existen en estos momentos en España 223 universidades federadas en 23 provincias y 11 comunidades autónomas que acogen aproximadamente a 91.800 participantes en diferentes actividades y programas impartidos por 3.200 profesionales de la educación popular 25 .

A estos colectivos se le aplicó un amplio cuestionario del que sólo destacamos aquí una pregunta referida al tiempo libre por ser coincidente con una de las preocupaciones más críticas de los planes gerontológicos.

Se trata de dos colectivos muy diferentes. El de las Universidades Populares está compuesto por un grupo más numeroso (216) en el cual 50 hombres, 162 mujeres y cuatro que no se identifican. Su nivel cultural es bajo ya que no tuvieron muchas oportunidades de formación. El colectivo de "IV ciclo" es numéricamente menor (un total de 79 personas con mayor equilibrio entre hombres [37], mujeres [39] y tres sujetos que no contestan). Su nivel de formación es medio-alto aunque ésta no sea una exigencia exclusiva para poder participar en dicha actividad de formación.

desarrollan sus programas: Vigo, Ourense, Cambados e Illa de Arousa. Recientemente se clausuró la de Vilanova de Arousa.

24. Las personas mayores cursan 27 créditos ( 9 créditos por curso: 90 horas) de distintas materias distribuidas en cuatro ámbitos de formación: Ciencias de la Salud; Ciencias Experimentales y Tecnología; Ciencias Sociales y Jurídicas y Humanidades. Al final de dicho período la universidad les ofrece un título propio de "Diplomado Senior".

25. Federación Española de Universidades Popllares (F.e.c.p.) (1999): Datos estadísticos. Actualización en 1999 con datos referidos al curso 1997-98. Madrid. 
AGUSTÍN REQUEJO OSORIO

PLANES GERONTOLÓGICOS Y PROYECTOS DE ANIMACIÓN SOCIOCLLTURAL PARA LAS PERSONAS MAYORES

TABLA № 2. Ocupaciones de ocio y tiempo libre de personas mayores

\begin{tabular}{|l|r|r|r|r|r|r|r|r|}
\hline & \multicolumn{3}{|c|}{ Universidades Populares } & \multicolumn{3}{c|}{ IV Ciclo Universitario } \\
\hline ACTIVIDADES & 1 & 2 & 3 & 4 & 1 & 2 & 3 & 4 \\
\hline Estar en casa sin hacer nada & 3,2 & 14,35 & 51,6 & 30,9 & 3,8 & 19,0 & 35,4 & 41,8 \\
Ir al bar, cafetería & 6,5 & 48,4 & 18,0 & 27,2 & 12,7 & 40,5 & 11,9 & 35,4 \\
Lectura (libros, periódicos...) & 67,3 & 18,4 & 4,6 & 9,7 & 83,5 & 7,6 & 0 & 8,9 \\
Reuniones con los amigos & 41,9 & 39,2 & 2,3 & 16,6 & 48,1 & 15,2 & 0 & 36,7 \\
Asistir a conferencias, conciertos & 26,3 & 45,2 & 9,7 & 16,9 & 40,5 & 41,8 & 0 & 17,7 \\
Ir al cine, al teatro & 12,9 & 38,7 & 22,6 & 25,8 & 11,4 & 45,6 & 6,3 & 36,7 \\
Ver la televisión & 45,6 & 30,4 & 0,9 & 23,0 & 46,8 & 26,6 & 2,5 & 24,1 \\
Escuchar la radio & 59,9 & 19,4 & 7,8 & 12,9 & 54,4 & 19,0 & 2,5 & 24,1 \\
Cuidar de los nietos & 16,1 & 11,1 & 11,1 & 61,3 & 12,7 & 10,1 & 12,7 & 64,6 \\
Pasear, caminar & 62,7 & 20,7 & 3,7 & 12,9 & 74,7 & 7,6 & 0 & 17,7 \\
Ir al club, hogar del pensionista & 2,8 & 4,6 & 37,8 & 54,8 & 2,5 & 1,3 & 45,6 & 50,6 \\
Otra actividad ${ }^{26}$ & 24,4 & 2,3 & 0,9 & 72,9 & 38,0 & 0 & 0 & 62,0 \\
Otra actividad & 15,9 & 0,9 & 0,9 & 82,5 & 8,9 & 2,5 & 0 & 88,6 \\
\hline
\end{tabular}

(1: Frecuentemente; 2: Ocasionalmente; 3: Nunca; 4: No contesta. La suma supone el 100\%).

A la vista de los resultados podemos constatar que por orden preferente, si nos atenemos a la primera categoría, el tiempo libre de estas personas mayores está ocupado en las siguientes actividades que realizan "frecuentemente": 1) Pasear y caminar: lo en hacen en uU.pp. el $62,7 \%$ de los sujetos y en el IV ciclo el 74,7\%. 2) Lectura (libros y periódicos): Uu.PP.: 67,3\%; IV ciclo: 83,5\%. 3) Escuchar la radio: Uu.PP.: 59,9\%; IV ciclo: 54,4\%. 5) Ver la televisión: Uu.PP.: 45,6\%; IV ciclo: 46,8\%. 6) Asistir a conferencias, conciertos: UU.PP.: 26,3\%; IV ciclo: $40,5 \%$ de los sujetos.

Estos datos nos señalan algunas características particulares de estos dos grupos. Hay una escasa diferencia entre ellos en cuanto a ver la televisión o escuchar la radio, pero lo hacen con menos frecuencia respecto a los datos aportados en los planes gerontológicos. En cuanto a las actividades de lectura y de conferencias son mucho menos frecuentes en el grupo de universidades populares que en el IV ciclo. Tal diferencia tiene su razón de ser por el distinto nivel de formación en este último grupo y debido a las facilidades que estos sujetos tienen por encontrarse en un ambiente universitario en el que se le ofrecen más facilidades para participar en este tipo de actividades.

Por último, la suma de las dos categorías (frecuentemente, ocasionalmente) ofrece diferencias menos importantes entre ambos grupos ocupando la lectura el primer lugar para el $87,7 \%$ de las personas mayores en UU.PP. y 91,1\% en IV ciclo

26. En el caso de "Otras" aparecen referencias a "labores domésticas" (particularmente para las mujeres); participación en reuniones (asociaciones, actividades sociales, políticas, etc.). 
universitario. Le siguen a continuación y por el mismo orden: "pasear, caminar" $(83,2 \% / 82,3 \%)$; asistir a conferencias para el Iv ciclo $(82,3 \%)$ frente a las reuniones con los amigos $(81,1 \%)$ para uU.PP. y $63,3 \%$ para el IV ciclo.

Finalmente en cuanto a la radio y televisión prevalece un mayor uso de la radio en ambos colectivos probablemente por el carácter de "acompañamiento" que tiene frente al carácter más absorbente" de la televisión. En todo caso es una actividad que se sitúa en el cuarto lugar (79,5\%) en los mayores de las universidades populares y en quinto lugar para el grupo del iv ciclo universitario $(73,4 \%)$.

En lo que ambos grupos también coinciden es en no frecuentar los clubs y/o hogares del pensionista donde se realizan otro tipo de actividades y programas de carácter "más pasivo" (7,4\% en el caso de uu.PP. y 3,8\% para IV ciclo universitario).

Si comparamos estos datos con los resultados que ofrece el Plan Gerontólogico de Madrid, por ser el que presenta cuestiones semejantes, podemos observar que la actividad que realizan más frecuentemente los mayores (en este caso "todos los días") es ver la televisión (93\%) seguida de oír la radio (77\%), pasear (70\%) y tareas domésticas (68\%). Muy lejos quedan las actividades relacionadas con la lectura tanto de periódicos (32\%) como de libros, revistas, etc. (28\%).

Todo ello quiere decir que aquellos programas que son diseñados de forma específica para mantener un estilo de vida de carácter más participativo y formativo cambian por completo las actividades de las personas mayores y transforman sus estilos de ocio. En los resultados de estos programas que evidentemente tienen un carácter más selectivo (sobre todo el iv ciclo universitario), el enfoque del tiempo libre es muy distinto del que con carácter general nos presentan las opiniones de los planes gerontológicos analizados en la primera parte.

Ello indica que ésta es la línea a seguir, tratando de implicar a las personas mayores en actividades que promuevan un ocio y un tiempo libre mucho más activo y formativo que contribuya a estilos de vida y de participación social más positivos para su realización personal.

\section{CONCLUSIONES}

Con carácter general podemos decir que el Plan Gerontológico Estatal y los Planes Gerontológicos de carácter autonómico tienen el mérito de poner de relieve el tema de las personas mayores en nuestra sociedad como un colectivo que merece particular atención.

Su aprobación y puesta en acción creemos que está ayudando a poner en marcha objetivos básicos enunciados para el año 2001 por la Asamblea Mundial para el Envejecimiento y por los respectivos planes gerontológicos.

El propio desarrollo de los planes en sus diversos apartados creo que estimula políticas y compromisos más activos a corto y medio plazo sobre aspectos tan fundamentales como el tema de pensiones; salud y asistencia sanitaria; servicios sociales; cultura y ocio y, finalmente, participación. 
En estas líneas nos hemos preocupado de entender y explicar el marco teórico de la política social con las personas mayores y analizar sus logros y deficiencias. Al mismo tiempo, hemos destacado las situaciones de cultura y ocio como tema más particular considerando los proyectos de animación sociocultural como la mejor propuesta de acción comunitaria para promover en las personas y en los grupos de mayores una actitud de participación activa en el proceso de su desarrollo personal y compromiso social.

Desde tal consideración se hace necesario cambiar la mentalidad sobre la situación de la "vejez" y reconceptualizar la intervención educativa y social con las personas mayores desde la perspectiva de "acciones de capacitación" frente a "acciones puntuales de asistencia" integrando los tres tipos de tiempo libre: el "tiempo libre experiencial" que aporta relax y diversión etc.; el "tiempo de formación" que aporta nuevas pautas de curiosidad y de interés por los problemas de hoy y, finalmente, el "tiempo social" que facilita el encuentro, las relaciones sociales y oportunidades de servicio a la sociedad.

Cuando esto es así mediante programas concretos, las actividades de ocio y tiempo libre cambian drásticamente en este colectivo. El puntual estudio empírico de dos colectivos de personas mayores que asisten a los programas de las universidades populares y del $\mathrm{IV}$ ciclo universitario nos muestra cómo el empleo de su tiempo de ocio tiene muy poco de parecido con el del colectivo de mayores que están ajenos a estas posibilidades formativas.

\section{BibliograFía}

AA.VV. (1995): Melanges Pierre Vellas. Recherches et réalisations. Pedone. París. Preface B. Saint Girons. Université de Toulouse.

A.I.U.T.A. (1997): L'apport des U.T.A. aux étudiants. L'apport des U.T.A. dans la societé 1996/97. Edup., Roma.

Alcalá Mangas, M. E. y Valenzuela Sánchez, R. (eds.): El aprendizaje de los mayores ante los retos del nuevo milenio. Ministerio de Trabajo y Asuntos Sociales. Dykinson, Madrid.

Bazo, M. T. (1999): Los mayores en Europa. Caja Madrid. Biblioteca Nueva, Madrid.

BeLanger, P. y FALGaS, R. M. a (1997): “La educación de adultos y las personas de edad. Tendencias y aspectos críticos". IIZ. DVV, 49, 181-184.

Castells, M.; Pérez Ortiz, L. y Guillemard, A. M. (1992): Análisis de las políticas de vejez en España en el contexto Europeo. Inserso, Madrid.

Comunidad De MAdRid (1998): Plan de Mayores. Consejería de Sanidad y Servicios, Consejería de Sanidad, Bienestar Social y Dirección General de Acción Social, Madrid.

DOCUMENTACIÓN SOCIAL (1998): "Las personas mayores». Cáritas Española, 112 (julio/septiembre).

Domínguez ANgulo, J. (1998): ¿Qué hacen las personas mayores por nosotros? Ediciones Delfín, Madrid.

DuChesne, P. (1997): L'Âge du Viellissement. Artel, Namur.

Dumazedier, J. (1971): Ocio y sociedad de clases. Fontanella, Barcelona.

(C) Ediciones Universidad de Salamanca

Teor. educ. 12,2000 , pp. 85-105 
ElizAZU, C. (1999): La Animación con personas mayores. Editorial ccs, Madrid.

EUROPEAN ASSOCIATION FOR THE EDUCATION OF ADULTS (1994): Older adults as belpers in learning process. E.A.E.A. Monografías, Barcelona.

Eurostat (1999): Europa en cifras. Mundi Prensa, Madrid

Federación Española de Untversidades Populares (1999): Datos estadísticos. Madrid.

Fermoso, P. (1998): “Políticas sociales y educación social en las 17 comunidades autónomas”. Politicas sociales y Educación Social. Grupo Editorial Interuniversitario, Granada, pp. 82-130.

Fundación EsPañola de la Tercera Edad (1999): Guia Nacional de Servicios Sociales para la Tercera Edad. Madrid.

García Carrasco, J. (coord.) (1997): Educación de Adultos. Ariel, Barcelona.

Generalitat de Catalunya (1995): Promoció dels drets i la vida activa de les persones grans: Pla integral de la Gent Gran. Noves Iniciatives i programes. Departament de Benestar Social, Barcelona.

Gobierno de Navarra (1997): Plan Gerontológico de Navarra. Modelo de acción. Pamplona.

Gobierno VAsco (1990): Plan Gerontológico de Euskadi. Servicio Central de Publicaciones del Gobierno Vasco, Vitoria.

Gómez Hernández, F. (1991): “Funcionamiento de las Universidades de la Tercera Edad en España como centros superiores de Educación de Adultos". A.I.U.T.A. (Actes). Université du Québec à Hull, Québec, pp. 348-358.

GuIRAO, M. y SÁNCHEZ, M. (1997): "Los programas universitarios para mayores en España". En LEMIEUX, A.: o. cit., pp. 145-157.

JunTA DE CASTILLA Y LEÓN (1995): Plan regional sectorial para personas mayores. Consejería de Sanidad, Bienestar Social y Dirección General de Acción Social, Valladolid.

Junta de COMunidades Castilla-la MANCHa (1998): Plan de atención a las personas mayores (1998-2002). Dirección General de Servicios Sociales, Toledo.

LEMIEUX, A. (1997): Los programas universitarios para mayores. Ministerio de Trabajo y Asuntos Sociales, Madrid.

Limon, M. ${ }^{a}$ R. (1993): "Tiempo de ocio. Tiempo de cultura en la Tercera Edad". Revista Educadores, 196, 235-354.

- (1995): "Acción de los organismos internacionales en la educación de la Tercera Edad". Revista Educadores, 236, 519-537.

Llull Peñalba, J. (1999): Teoría y práctica de la educación en el tiempo libre. Editorial ccs. Madrid, pp. 20 y ss.

LuMSDEN, D. B. (1984): The older adult as learner. Hemisphere Publishing Corporation, Washington.

Mayán Santos, J. M. (1997): Cuarto Ciclo Universitario: Alumnos mayores de 55 años. Universidad de Santiago de Compostela.

MidWINTER, E. (1998): Leisure: New Opportunities in the third age. Centurion Press Limited, London.

Miguel, J. A. (1998): "Balance de las políticas sociales con los mayores en España. Los planes gerontológicos. Análisis y perspectivas". Documentación Social, 112, 175-191.

Ministerio de Asuntos Sociales (1992): Plan Gerontológico. Madrid.

Peña Calvo, J. V.; García del Dujo, A.; Sánchez Valle, I. y Vera Vila, J. (2000): "La medida de la calidad de vida en el proceso de revisión de las políticas de bienestar". XIX Seminario Interuniversitario de Teoría de la Educación. San Lorenzo de El Escorial.

REQuejo OsORIO, A. (1997): "Animación sociocultural y Educación de Adultos" y "Animación sociocultural en la Tercera Edad». En Trilla, J.: o. cit., pp. 239-268. 
- (1999): "Educación de adultos: Programas Universitarios para personas Mayores". Revista ADAXE. Revista de Estudios y Experiencias Educativas. Universidad de Santiago de Compostela.

Seminario de Pedagogía Social (1998): "Políticas sociales y Educación social para la Tercera Edad". Actas del XII Seminario de Pedagogía Social. Editorial Interuniversitaria, Granada. Trilla, J. (1993): Otras educaciones. Animación Sociocultural, formación de adultos y ciudad educativa. Anthropos, Barcelona, p. 93.

- (1997) (coord.): Animación sociocultural: Teorias, programas y ámbitos. Ariel, Barcelona. 